\title{
Chemokine-mediated recruitment of NK cells is a critical host defense mechanism in invasive aspergillosis
}

\author{
Brad E. Morrison, ${ }^{1}$ Stacy J. Park, ${ }^{1}$ Jill M. Mooney, ${ }^{2}$ and Borna Mehrad ${ }^{1}$ \\ ${ }^{1}$ Department of Medicine, Division of Pulmonary and Critical Care Medicine, and \\ ${ }^{2}$ Department of Pathology and the Immunology Graduate Program, University of Texas Southwestern Medical Center, \\ Dallas, Texas, USA
}

\begin{abstract}
Invasive aspergillosis is a severe pneumonia that is usually fatal despite currently available therapy. The disease disproportionately afflicts immunocompromised patients, indicating the critical importance of the immune status of the host in this infection, but the defense mechanisms against this pathogen remain incompletely understood. In the current study, we hypothesized that the chemokine ligand monocyte chemotactic protein-1, also designated CC chemokine ligand-2 (MCP-1/ CCL2) is necessary for effective host defense against invasive aspergillosis in immunocompromised hosts. We found a rapid and marked induction of MCP-1/CCL2 in the lungs of neutropenic mice with invasive aspergillosis. Neutralizing MCP-1/CCL2 resulted in twofold greater mortality and greater than threefold increase in pathogen burden in the lungs. Neutralization of MCP-1/CCL2 also resulted in reduced recruitment of NK cells to the lungs at early time points, but did not affect the number of other leukocyte effector cells in the lungs. Ab-mediated depletion of NK cells similarly resulted in impaired defenses against the infection, resulting in a greater than twofold increase in mortality and impaired clearance of the pathogen from the lungs. These data establish MCP-1/ CCL2-mediated recruitment of NK cells to the lungs as a critical early host defense mechanism in invasive aspergillosis and demonstrate NK cells to be an important and previously unrecognized effector cell in this infection.
\end{abstract}

J. Clin. Invest. 112:1862-1870 (2003). doi:10.1172/JCI200318125.

\begin{abstract}
Introduction
Invasive aspergillosis is an infection characterized by a rapidly increasing incidence and remarkably high case-fatality rate, even with appropriate therapy $(1,2)$. The fungal spores, known as conidia, are ubiquitously distributed in both indoor and outdoor air and are deposited deep in the lungs when inhaled. Conidia are efficiently cleared from the lungs in healthy people, but in immunocompromised patients they can germinate to form hyphae that invade the surrounding tissues, resulting in a severe and progressive pneumonia that can subsequently disseminate to other organs. Since normal innate immune responses are almost always sufficient to contain the pathogen, understanding the host defense mechanisms in this infection is of interest and may lead to the development of new therapies.
\end{abstract}

Received for publication February 13, 2003, and accepted in revised form October 21, 2003.

Address correspondence to: Borna Mehrad, University of Texas Southwestern Medical Center, 5323 Harry Hines Boulevard, Dallas, Texas 75390-9034, USA. Phone: (214) 648-3429;

Fax: (214) 648-3545;

E-mail: Borna.Mehrad@UTSouthwestern.edu.

Conflict of interest: The authors have declared that no conflict of interest exists.

Nonstandard abbreviations used: monocyte chemotactic protein-1, also designated CC chemokine ligand-2 (MCP-1/CCL2); Gomori methanamine silver (GMS); myeloperoxidase (MPO); phycoerythrin (PE); polymorphonuclear (PMN).
Influx of effector leukocytes is a critical event in the lung's innate immune response to pathogens. The local production of chemotactic molecules, including chemokine ligands, is essential in orchestrating the recruitment of these effector leukocytes to the site of infection. While it is likely that most chemokine ligands and receptors have now been discovered (3), the role of these mediators in the complex signaling that occurs in the setting of specific disease states remains to be elucidated (4). Monocyte chemotactic protein-1, also designated CC chemokine ligand-2 (MCP-1/ CCL2) is a chemokine ligand that mediates a remarkably diverse set of effects in different disease models; for example, it mediates Th1 polarization in cryptococcal pneumonia $(5,6)$, airway Th2-acquired immunity to Aspergillus and OVA (7-9), pulmonary fibrosis (10), and experimental allergic encephalitis (11). These divergent effects underscore the role of this mediator in multiple facets of both normal and pathological immune responses.

Clinically, reduced numbers or impaired function of neutrophils are by far the best-characterized risk factors for invasive aspergillosis (12-15), but most patients with invasive aspergillosis have multiple and incompletely understood defects in antimicrobial host defenses. NK cells are a population of effector leukocytes that, unlike most other lymphocyte populations, do not express clonally preserved antigen receptors and thus do not require clonal proliferation before response to antigenic challenges. The role of NK cells in the con- 
text of innate immunity is well characterized in response to tumor cells, transplanted cells, and several classes of pathogens, including many viruses (16) and intracellular bacteria $(17,18)$. The role of NK cells in the innate immune response against fungi has not been studied in detail, however.

In a previous study, we found an association between reduced induction of MCP-1/CCL2 in the lungs and more severe invasive aspergillosis in mice (19). We thus hypothesized that MCP-1/CCL2 is necessary for effective host defense against invasive aspergillosis in neutropenic hosts and that its depletion results in worsened outcome of the infection. The data presented here link MCP-1/CCL2 production to the recruitment of NK cells to the lungs and provide evidence for a previously unrecognized, NK cell-mediated, innate immune mechanism in neutropenic hosts with invasive aspergillosis.

\section{Methods}

Animals. WT 8- to 12-week-old age- and sex-matched $\mathrm{C} 57 \mathrm{BL} / 6$ and BALB/c mice (The Jackson Laboratories, Bar Harbor, Maine, USA) and CCR2-/- mice backcrossed for eight generations onto BALB/c background (20), a generous gift from Galen B. Toews (University of Michigan, Ann Arbor, Michigan, USA), were bred and maintained under specific pathogenfree conditions in the animal care facility at the University of Texas Southwestern Medical Center until the day of sacrifice.

In vivo immuno-neutralization and Ab-mediated cell depletion. Monoclonal anti-murine MCP-1/CCL2 Ab (2H5) was used for in vivo neutralization experiments $(5,21)$. The $A b$ was purified from a hybridoma supernatant (a generous gift from Martin E. Dorf, Harvard University, Cambridge, Massachusetts, USA) over a protein A column, according to the manufacturer's instructions (Amersham Pharmacia Biotech, Piscataway, New Jersey, USA). The buffer was exchanged for PBS using a PD10 column (Amersham Pharmacia Biotech), IgG concentration was determined by OD, and MCP-1/CCL2-neutralizing activity was determined by ELISA. In neutralization experiments, 200 $\mu \mathrm{g}$ anti-murine MCP-1/CCL2 Ab or PBS was administered intraperitoneally 2 hours before Aspergillus fumigatus inoculation, and $100 \mu \mathrm{g}$ was given on days 2 and 4 after inoculation. The mAb's were used for in vivo depletion of neutrophils (RB6-8C5, anti-Ly6G) $(22-24)$ and NK cells (PK136, anti-NK1.1) $(25,26)$ in different experiments. These mAb's were produced by intraperitoneal injection of hybridoma into nude mice and ascites collection (performed by TSD BioServices, Germantown, New York, USA); the concentration of IgG in the pooled ascitic fluid was quantified by HPLC. In preliminary studies, administration of $100 \mu \mathrm{l}$ of a 1:200 dilution of RB6-8C5 ascites intraperitoneally 1 day before A. fumigatus challenge resulted in peripheral blood neutropenia (absolute circulating neutrophil count less than 50 cells/ $\mu \mathrm{l}$ ) on days 1 and 3 after $A b$ administration in both infected and control animals, with a return of peripheral counts to pretreatment levels by day 5 . Concomitantly, neutrophils accumulate in the lungs of infected animals in association with areas of hyphal invasion by day 3 after Aspergillus challenge. Administration of RB6-8C5 to uninfected mice resulted in no detectable alteration in non-neutrophil leukocyte populations in blood or examined organs (data not shown). Intraperitoneal injection of $200 \mu \mathrm{g}$ of anti-NK1.1 2 days before A. fumigatus challenge resulted in depletion of splenic NK cells for 5 days. Administration of irrelevant $A b$ 's, either alone or in combination with neutrophil depletion, had no detectable effect on clearing the pathogen (data not shown).

Preparation and administration of A. fumigatus conidia. A mouse model of invasive aspergillosis using A. fumigatus strain 13073 (American Type Culture Collection, Rockville, Maryland, USA), which was used in all present studies, has been characterized previously $(19,24$, 27). The organism was grown on Sabouraud dextrose agar plates (Becton Dickinson Microbiology Systems, Cockeyville, Maryland, USA) for $7-10$ days at $37^{\circ} \mathrm{C}$. The plate surface was washed with sterile $0.1 \%$ Tween80 (Sigma-Aldrich, St. Louis, Missouri, USA) in PBS, and the resulting conidial suspension was filtered through sterile gauze to remove clumps and hyphal debris. The concentration of conidia was determined using a hemacytometer, the suspension was diluted to the desired concentration, and the concentration was again measured before administration. In preliminary experiments, the number of particles determined using the hemacytometer was in close agreement with the number of viable CFUs found by serial dilution and culture of the suspension (data not shown). On the day of inoculation, animals were anesthetized with a cocktail of ketamine and xylazine administered intraperitoneally. The trachea was exposed using standard aseptic technique, $30 \mu \mathrm{l}$ inoculum (A. fumigatus suspension or $0.1 \%$ Tween- 80 vehicle) was administered by a sterile 26-gauge needle, and the skin was closed with surgical staples. Animals were challenged with inocula ranging from $2 \times 10^{6}$ to $3 \times 10^{6}$ conidia in various experiments. In preliminary studies, intratracheal administration of vehicle alone did not result in changes in histology or influx of neutrophils (data not shown).

Lung harvest. At designated time points, mice were sacrificed by $\mathrm{CO}_{2}$ asphyxiation, and chest cavities were opened aseptically. For histologic examinations, lungs were perfused, inflated, fixed in $4 \%$ paraformaldehyde in PBS, and processed and stained with $\mathrm{H} \& \mathrm{E}$ or Gomori methanamine silver (GMS) stains. For the various assays, lung vasculature was perfused with $1 \mathrm{ml}$ PBS containing $5 \mathrm{mM}$ EDTA through the right ventricle, excised, frozen in liquid nitrogen, and stored at $-20^{\circ} \mathrm{C}$ until the day of the assay. On the day of the assay, samples were homogenized in complete protease inhibitor cocktail buffer (Roche Applied Science, Indianapolis, Indiana, USA) in PBS, sonicated, and the 


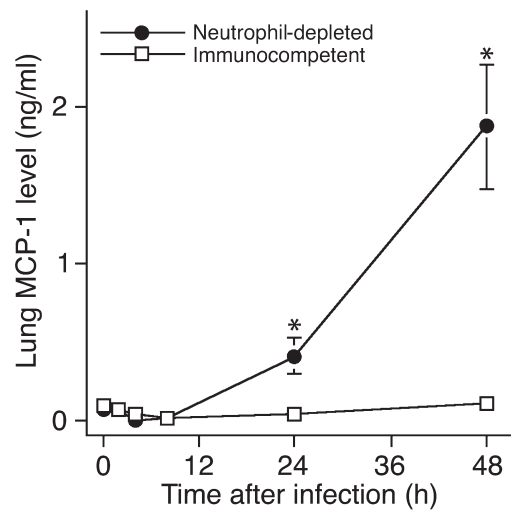

Figure 1

Lung MCP-1/CCL2 levels after intratracheal $A$. fumigatus challenge in immunocompetent and neutropenic animals. Both groups were inoculated intratracheally with conidia, and MCP-1/CCL2 was measured in lung homogenates using a specific ELISA. ${ }^{*} P<0.05$ compared with immunocompetent animals at that time point. Data shown represent mean $\pm \mathrm{SEM} ; n=6$ for each group at each time point. Neutrophildepleted, RB6-8C5 mAb-treated animals.

resulting slurry used to assay myeloperoxidase (MPO) activity or chitin content. For ELISAs, the slurry was pelleted, and supernatants were passed through 0.45$\mu \mathrm{m}$ filters (Gelman Sciences Inc., Ann Arbor, Michigan, USA) and stored at $4^{\circ} \mathrm{C}$ for up to 3 days, until ELISA was performed using a MCP-1/CCL2-specific kit according to the manufacturer's instructions $(R \& D$ Systems Inc., Minneapolis, Minnesota, USA; minimum detectable concentration less than $2 \mathrm{pg} / \mathrm{ml}$ ).

Lung MPO activity. Organ MPO activity was measured as a marker of neutrophil sequestration, as described previously (28). Briefly, a $100 \mu \mathrm{l}$ aliquot of each lung homogenate was added to $100 \mu \mathrm{l}$ of a buffer containing $50 \mathrm{mM}$ potassium phosphate $(\mathrm{pH} 6.0), 5 \%$ hexadecyltrimthylammonium bromide, and 5 mM EDTA. Samples were sonicated for 10 seconds and centrifuged at $3,000 \mathrm{~g}$ for 15 minutes. The supernatant was mixed at a ratio of $1: 15$ with a buffer containing $1 \mathrm{M}$ monobasic potassium phosphate, $1 \mathrm{M}$ dibasic potassium phosphate, $3 \% \mathrm{H}_{2} \mathrm{O}_{2}$, and $o$-dianisidine hydrochloride, and read at $490 \mathrm{~nm}$. MPO activity was calculated as the change in absorbency over time.

Lung chitin assay. Molds, such as Aspergillus species, grow as multicellular branching filamentous structures (hyphae) and do not reliably form reproductive structures (conidia) in infected tissues (29). As a result, serial dilution and culture of homogenized tissue is not an adequate measure of pathogen burden in these infections. We therefore used a previously described assay for chitin in order to quantify the burden of the pathogen in the lungs (19). Chitin, a component of the hyphal wall, is absent from conidia and vertebrate tissues. The level of chitin, as detected by this assay, correlates directly with the weight of hyphae (30). Lungs were homogenized, centrifuged, and resuspended in sodium lauryl sulfate $(3 \% \mathrm{wt} / \mathrm{vol})$, heated at $100^{\circ} \mathrm{C}$ for
15 minutes, washed, and resuspended in $\mathrm{KOH}(120 \%$ $\mathrm{wt} / \mathrm{vol})$. It was then heated at $130^{\circ} \mathrm{C}$ for 60 minutes, cooled, incubated on ice after the addition of $8 \mathrm{ml}$ of ice-cold $75 \%$ ethanol, pelleted after the addition of 0.3 $\mathrm{ml}$ Celite (diatomaceous earth) suspension, washed, and resuspended in $0.5 \mathrm{ml}$ distilled water. We added 0.5 $\mathrm{ml}$ of $\mathrm{NaNO}_{2}(5 \% \mathrm{wt} / \mathrm{vol})$ and $\mathrm{KHSO}_{4}(5 \% \mathrm{wt} / \mathrm{vol})$ to each sample and to standards that contained distilled water and $10 \mu \mathrm{g} / \mathrm{ml}$ glucosamine. Aliquots $(0.6 \mathrm{ml})$ of each were transferred to separate tubes, $0.2 \mathrm{ml}$ ammonium sulfamate and $0.2 \mathrm{ml}$ of 3-methyl-2-thiazolone hydrazone $\mathrm{HCl}$ monohydrate $(50 \mathrm{mg}$ in $10 \mathrm{ml}$ distilled water) were added, and samples were heated to $100^{\circ} \mathrm{C}$ for 3 minutes and then cooled. $\mathrm{FeCl}_{3} \cdot 6 \mathrm{H}_{2} \mathrm{O}(0.2 \mathrm{ml}$; $0.83 \% \mathrm{wt} / \mathrm{vol}$ ) was then added to each sample, and OD was measured at $650 \mathrm{~nm}$ after 25 minutes. Chitin content, in glucosamine equivalents, was then calculated: chitin content $=[5 \times(\mathrm{OD}$ of organ $-\mathrm{OD}$ of control organ)]: (OD of glucosamine - OD of water).

Lung single-cell suspension. Single-cell suspensions of the lungs were prepared as previously described (31). Briefly, perfused lungs were resected, minced to a fine slurry, and incubated in $1 \mathrm{mg} / \mathrm{ml}$ type A collagenase (Roche Applied Science) and $30 \mathrm{U} / \mathrm{ml}$ DNase (SigmaAldrich) in RPMI (Sigma-Aldrich) at $37^{\circ} \mathrm{C}$ for $30 \mathrm{~min}$ utes. Cells were then mechanically dispersed by aspirating and ejecting the slurry in syringes (Becton Dickinson and Co., Franklin Lakes, New Jersey, USA), passed through Nitex mesh filters (Tetko Inc., Kansas City, Missouri, USA), and then passed through a $40 \%$ Percoll gradient (Sigma-Aldrich) before cell counting under a hemacytometer.

Flow cytometry. For identification of various leukocyte populations, $10^{6}$ cells from lung or spleen suspensions, prepared as described above, were stained for $15 \mathrm{~min}$ utes on ice with the following Ab's: anti-CD3-allophycocyanin (anti-CD3-APC), anti-CD8-phycoerythrin (anti-CD8-PE), anti-CD11b-APC, anti-CD45-peridinin chlorophyll A protein (anti-CD45-PerCP), antiDX5-PE or -FITC, anti-F4/80-PE, anti-Ly6G-FITC, and anti-NK1.1-biotin or -PE (all from PharMingen, San Diego, California, USA). Samples were then washed in staining buffer, fixed in 1\% paraformaldehyde (Sigma-Aldrich) in PBS, and kept in the dark at $4^{\circ} \mathrm{C}$ until analyzed on a FACScalibur cytometer using Cellquest software (Becton Dickinson Immunocytometry Systems, San Jose, California, USA). Absolute numbers of leukocyte subsets were calculated as the product of the percentage of each cell type and the total number of cells in the sample.

Isolation, culture, and adoptive transfer of NK cells. Splenocytes were cultured at $3 \times 10^{6} \mathrm{cells} / \mathrm{ml}$ in DMEM with $10 \%$ FCS, antibiotics, nonessential amino acids, indomethacin $(0.5 \mu \mathrm{g} / \mathrm{ml}), 2$-mercaptoethanol $(2.5$ $\mu \mathrm{M})$, murine IL-12 (mIL-12, $1 \mathrm{ng} / \mathrm{ml})$, and $\mathrm{mIL}-18$ $(100 \mathrm{ng} / \mathrm{ml})$ for 5 days. Cultured NK cells were then enriched by depletion of $\mathrm{CD}^{+}$, $\mathrm{Ly}-6 \mathrm{G}^{+}$, TER- $119^{+}$, $\mathrm{CD} 22^{+}$, and $\mathrm{F} 4 / 80^{+}$cells using magnetic particles according to the manufacturer's instructions (Stem 

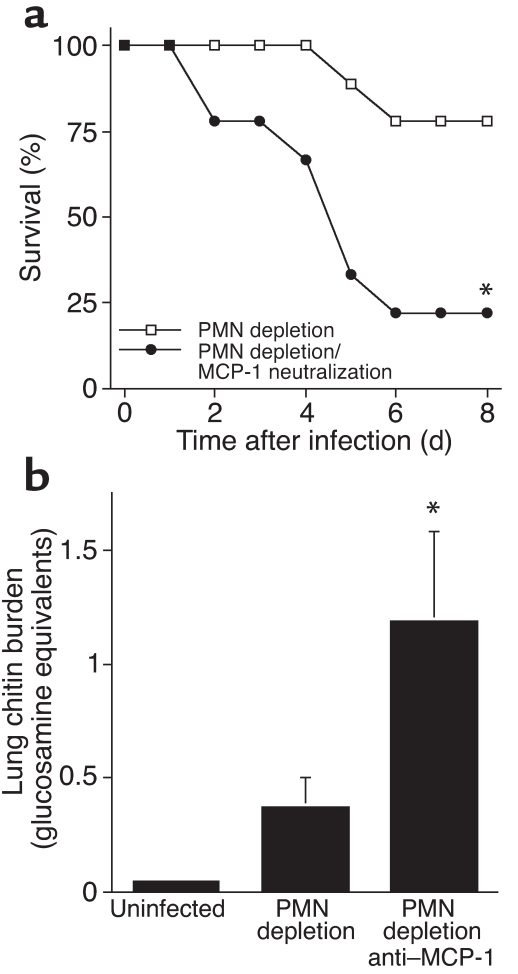

Cell Technologies Inc., Vancouver, British Columbia, Canada). In preliminary studies, eluted cells were approximately $95 \% \mathrm{DX}^{+}$(pan-NK cell marker) and $\mathrm{CD}^{-}$. In some experiments, cells were then stained with the vital cytoplasmic dye, CFSE (Molecular Probes Inc., Eugene, Oregon, USA), by incubating in 5 $\mu \mathrm{M}$ CFSE in PBS with 5\% FCS for 10 minutes and washing three times. Uniform staining of cells was verified by epifluorescent microscopy before adoptive transfer of $2 \times 10^{6}$ cells in $100 \mu \mathrm{l}$ PBS through a lateral tail vein on the day of inoculation.

Statistical analysis. Data were analyzed using a Macintosh G3 Powerbook computer and statistics software (InStat, version 2.01; GraphPad Software for Science

\section{Figure 2}

Outcome of invasive aspergillosis in neutropenic mice with or without neutralization of MCP-1/CCL2. (a) Survival study; $n=15$ for each group. (b) Lung chitin content after intratracheal $A$. fumigatus challenge on day 3 after intratracheal inoculation with conidia. Data represent mean \pm SEM of $n=6$ for each group. ${ }^{*} P<0.05$ compared with infected mice with polymorphonuclear (PMN) cell depletion. PMN depletion indicates mice treated with RB6-8C5 and PBS; PMN depletion/MCP-1 neutralization indicates mice treated with RB6$8 \mathrm{C} 5$ and anti-MCP-1/CCL2 mAb.

Inc., San Diego, California, USA). Survival data were compared using Fisher's exact test. All other data were expressed as mean plus or minus SEM and compared using an unpaired two-tailed Mann-Whitney (nonparametric) test. $P$ values were considered statistically significant if they were less than 0.05 .

\section{Results}

Production of MCP-1/CCL2 in the lung during invasive aspergillosis and effect of immuno-neutralization on outcome of the infection. We began by measuring the concentration of MCP-1/CCL2 protein in the lungs of normal and transiently neutrophil-depleted mice that had been challenged intratracheally with $A$. fumigatus conidia. We found a marked induction in lung MCP-1/CCL2 levels in response to the pathogen at 24 and 48 hours after inoculation in transiently neutropenic animals, but no measurable increase in immunocompetent animals (Figure 1). MCP-1/CCL2 remained undetectable in serum from both groups, suggesting a compartmentalized response limited to the lungs.

To assess whether MCP-1/CCL2 is required for host defense in invasive aspergillosis, we next examined the outcome of the infection in the setting of Ab-mediated neutralization of the ligand. In neutrophil-depleted mice with invasive aspergillosis, neutralization of MCP-1/CCL2 more than doubled the lethality of infection, as compared with infected neutrophildepleted mice treated with PBS (Figure 2a). We also

\section{Figure 3}

Effect of MCP-1/CCL2 neutralization on lung leukocyte subsets in transiently neutropenic mice after intratracheal challenge with $A$. fumigatus conidia. Cell populations were identified as $\mathrm{CD} 45^{+}$cells expressing the markers in parentheses. ${ }^{*} P<0.05$ compared with infected mice with $\mathrm{PMN}$ depletion at the same time point. Data shown represent mean $\pm \mathrm{SEM} ; n=4-6$ for each group at each time point. Uninfected indicates normal uninfected mice; infected/PBS indicates mice treated with RB6-8C5 and PBS and challenged with intratracheal $A$. fumigatus conidia; infected/anti-MCP-1 indicates mice treated with RB6-8C5 and anti-MCP-1/CCL2 $\mathrm{mAb}$ and challenged with intratracheal $A$. fumigatus conidia.
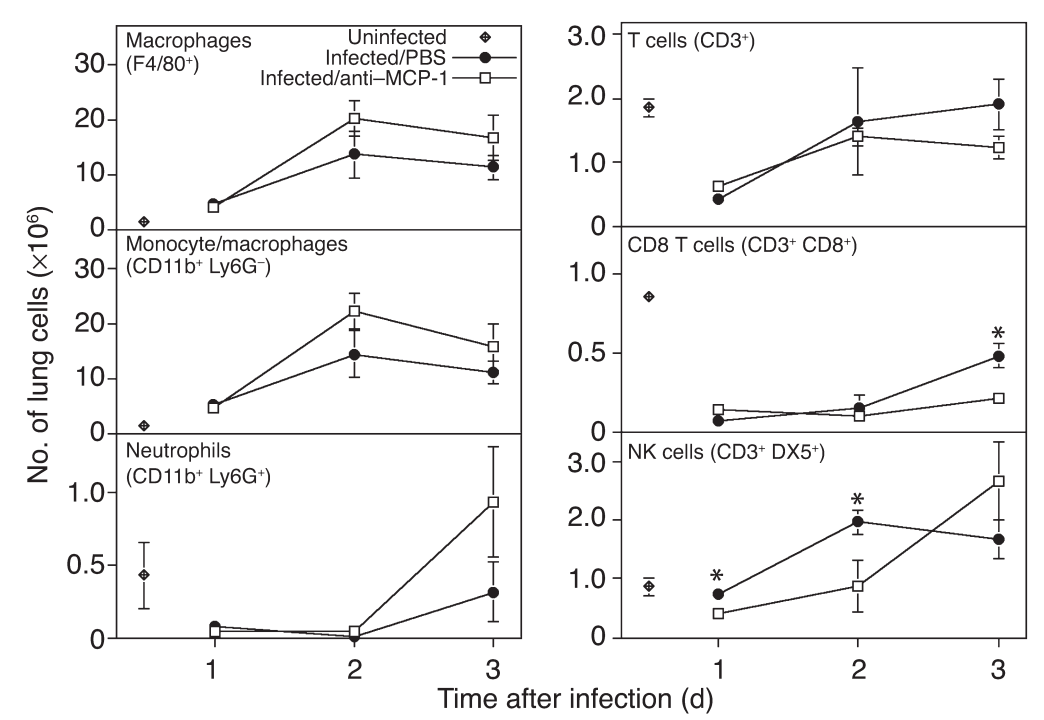


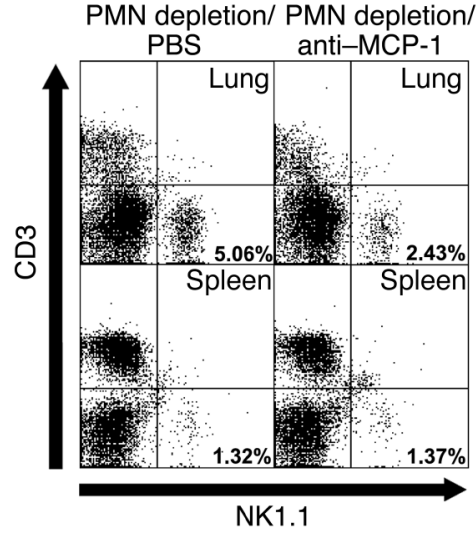

Figure 4

Effect of MCP-1/CCL2 neutralization on NK cells in neutropenic mice on day 1 after intratracheal challenge with $A$. fumigatus conidia. Flow-cytometry data gated on $\mathrm{CD} 45^{+}$cells with lymphocyte lightscatter characteristics. Data are representative of two experiments, with a total of five animals per group. PMN depletion/PBS indicates animals treated with RB6-8C5 mAb and PBS and challenged with intratracheal $A$. fumigatus conidia; PMN depletion/anti-MCP-1 indicates animals treated with RB6-8C5 and anti-MCP-1/CCL2 mAb's and challenged with intratracheal $A$. fumigatus conidia.

compared the fungal burden in the lungs between the two groups on day 3 after Aspergillus inoculation (Figure $2 \mathrm{~b}$ ). There was greater than threefold higher lung chitin content in the setting of MCP-1 neutralization as compared with mice with invasive aspergillosis treated with PBS, indicating a more severe infection in the setting of MCP-1/CCL2 neutralization.

Effect of neutralization of MCP-1/CCL2 on the influx of lenkocytes into the lungs in invasive aspergillosis. To evaluate the mechanism of action of MCP-1/CCL2 in host defense in this infection, we next quantified lung effector leukocyte subsets at various time points after challenging neutropenic mice with Aspergillus with or without MCP-1/CCL2 neutralization. On days 1 and 2 of infection, MCP-1/CCL2 neutralization did not affect the number of lung monocyte/macrophages, neutrophils, or $\mathrm{T}$ cells. We noted a marked reduction in numbers of total and $\mathrm{CD} 8^{+}$lung $\mathrm{T}$ cells after adminis-

\section{Figure 5}

Role of MCP-1/CCL2 in in vivo recruitment of NK cells to the lungs in invasive aspergillosis. Cultured NK cells were left unlabeled or labeled with the vital cytoplasmic fluorochrome, CFSE, and transferred to neutrophil-depleted mice before intratracheal challenge with $A$. fumigatus conidia or vehicle. Infected mice were treated with PBS or neutralizing anti-MCP-1/CCL2 Ab, and lungs were examined for the presence of CFSE-labeled cells after 1 day. (a) A representative scatter diagram of total lung cells, demonstrating the gate used in b. (b) Representative scatter diagrams from each group, gated on lung CD3- NK1.1+ cells in a. The upper-right quadrants contain transferred $\mathrm{CFSE}^{+} \mathrm{NK}$ cell populations used for statistical comparisons in c. (c) Mean \pm SEM of the number of lung $\mathrm{CFSE}^{+} \mathrm{CD}^{-}$NK cells in each group ( $n=3-5$ per group). ${ }^{*} P<0.05$ compared with uninfected mice given labeled cells; ${ }^{*} P<0.05$ compared with infected mice with PMN depletion. tration of conidia, compatible with previous literature demonstrating lung $\mathrm{T}$ cell apoptosis after intrapulmonary challenge with particulate antigens $(32,33)$. Conversely, we observed an early increase in the number of lung classical (CD3-) NK cells in neutrophildepleted mice, which was abrogated in the setting of MCP-1/CCL2 neutralization (Figure 3). Furthermore, we found reduced numbers of $\mathrm{NK}\left(\mathrm{CD}^{-}, \mathrm{NK} 1.1^{+}\right)$cells in the lungs, but not in the spleens, of mice with MCP1/CCL2 neutralization (Figure 4), suggesting an impairment of influx, rather than depletion of available cells, as the mechanism for the reduced number of lung NK cells in this setting.

We reasoned that the number of lung NK cells represents a balance between cell influx on the one hand and efflux or apoptosis on the other. We therefore sought to examine directly whether MCP-1/CCL2 mediates the early influx of NK cells into the lung in invasive aspergillosis. We transferred live, unlabeled, or CFSE-labeled NK cells intravenously to three groups of mice: uninfected neutrophil-depleted mice, infected neutrophil-depleted mice, and infected neutrophil-depleted mice with MCP-1/CCL2 neutralization (Figure 5). To exclude any nonspecific "trapping" of the intravenously transferred cells in the pulmonary blood vessels in areas of ongoing inflammation, we administered the labeled cells to recipients 30 minutes before intratracheal challenge with conidia. We found greater than eightfold more CFSElabeled cells in the lungs of infected mice, as compared with uninfected mice, suggesting that transferred cells entered the lung in the setting of infection. Importantly, the number of transferred NK cells in infected lungs was halved in the setting of MCP-1/CCL2 neutralization, suggesting that MCP-1/ CCL2 is necessary for early recruitment of NK cells to the lungs in this infection.

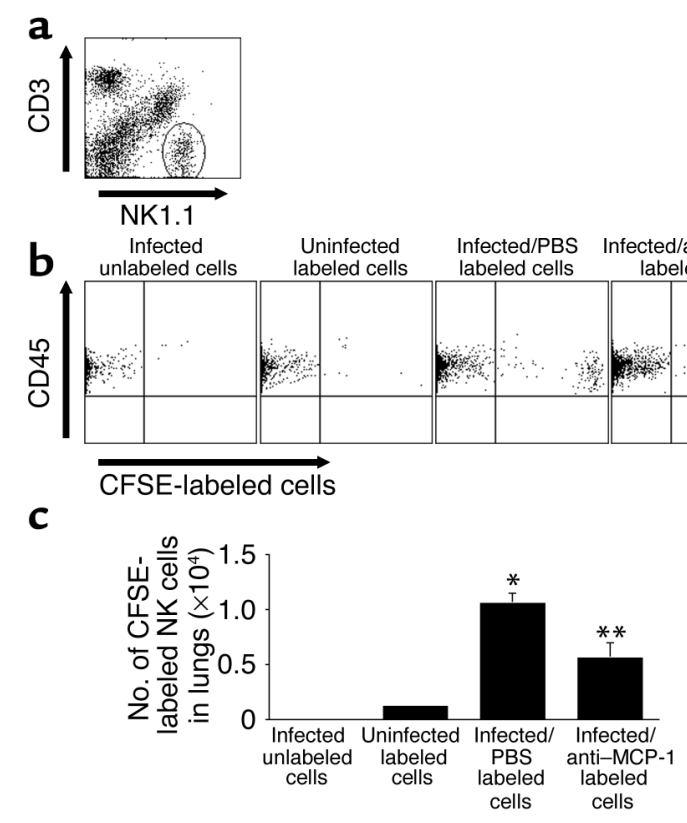


a

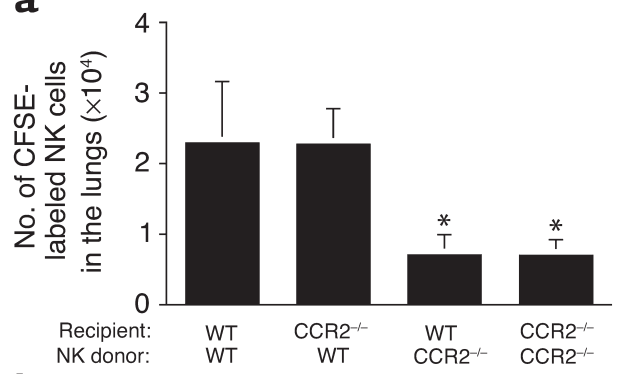

b

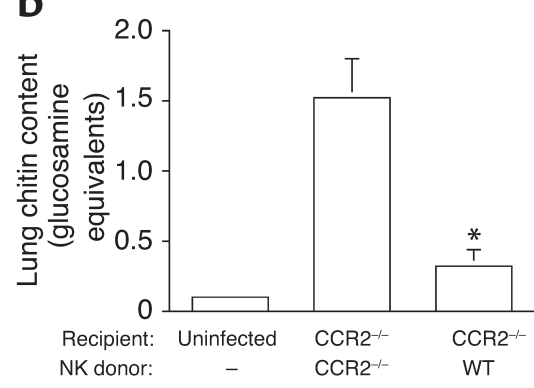

C

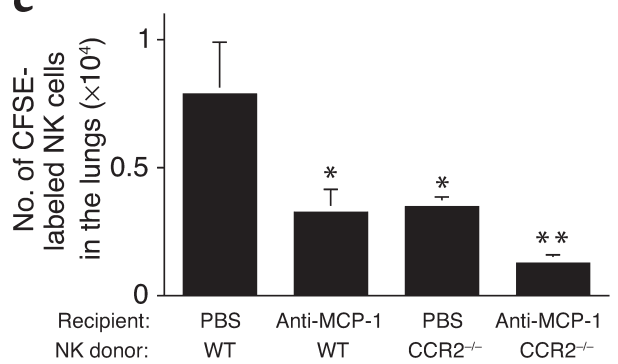

Role of CCR2 in lung NK cell influx and outcome of infection. Because CCR2, the only known signaling receptor for MCP-1/CCL2, is expressed on NK cells (34-39), we sought to corroborate our findings by examining the role of CCR2 on the recruitment of NK cells to the lung in this infection. We first injected CFSE-labeled NK cells from either WT or CCR2-/animals into neutrophil-depleted WT or CCR2-/mice before inoculation with $A$. fumigatus (Figure 6a). In both WT and CCR2-deficient recipients, there was approximately $70 \%$ fewer CFSE-labeled NK cells in the lungs of mice receiving CCR2-/- cells, as compared with WT cells. This indicates that NK cell expression of CCR2, the receptor for MCP-1/CCL2, is necessary for the influx of NK cells into the lungs in the setting of invasive aspergillosis. To assess whether CCR2-mediated influx of NK cells is impor-

\section{Figure 7}

Survival after intratracheal $A$. fumigatus challenge in neutropenic mice with or without NK cell depletion. (a) Survival study; $n=15$ for each group. ${ }^{*} P<0.05$. (b) Lung chitin content and MPO activity on day 3 after intratracheal inoculation with conidia in neutropenic mice. $\mathrm{MPO}$ activity is a surrogate for presence of neutrophils in the lung in this model. Data represent mean $\pm \mathrm{SEM} ; n=6$ for each group. PMN depletion indicates mice treated with RB6-8C5 and PBS; PMN and NK depletion indicates mice treated with RB6-8C5 and NK1.1.

\section{Figure 6}

Role of CCR2 in in vivo recruitment of NK cells to the lungs in invasive aspergillosis. CFSE-labeled or unlabeled NK cells from WT or CCR2 ${ }^{-/-}$animals were transferred to neutrophil-depleted mice before intratracheal challenge with $A$. fumigatus conidia. Infected mice were treated with PBS or neutralizing anti-MCP-1/CCL2 Ab. (a) Mean \pm SEM of number of lung CFSE ${ }^{+}$CD3 - DX $^{+}$NK cells $(n=3-5$ mice per group). ${ }^{*} P<0.05$ compared with corresponding recipients of WT NK cells. (b) Lung chitin content on day 3 of invasive aspergillosis after NK cell transfer. Mean \pm SEM ( $n=6$ mice per infected groups; $n=2$, uninfected group). ${ }^{*} P<0.05$ compared with recipients of $\mathrm{CCR}^{-/-} \mathrm{NK}$ cells. (c) Mean $\pm \mathrm{SEM}$ of number of lung $\mathrm{CFSE}^{+} \mathrm{CD} 3$ $\mathrm{DX} 5^{+} \mathrm{NK}$ cells in each group ( $n=4-6$ mice per group). Anti-MCP- 1 , mice treated with anti-MCP-1/CCL2 mAb. ${ }^{*} P<0.05$ compared with WT recipients of WT NK cells; ${ }^{*} P<0.05$ compared with each of the other groups.

tant to host defense in invasive aspergillosis, we next compared the lung fungal burden in CCR2 $2^{-/}$hosts with invasive aspergillosis injected with NK cells from either WT or CCR2 $2^{-/-}$hosts. CCR2 $2^{-/-}$mice that received WT NK cells had approximately $80 \%$ lower lung chitin content, suggesting that CCR2 expression on NK cells is important to combat fungal growth in this infection.

Our data indicated that MCP-1/CCL2 in the host and CCR2 expression on NK cells are each required for influx of $\mathrm{NK}$ cells to the lungs in invasive aspergillosis. We therefore sought to determine if influx of NK cells into the lungs was mediated by the exclusive interaction of this single ligand and single receptor. We hypothesized that MCP-1/CCL2induced NK cell influx was mediated solely by CCR2 expressed on NK cells and that the only role of NK cell

a
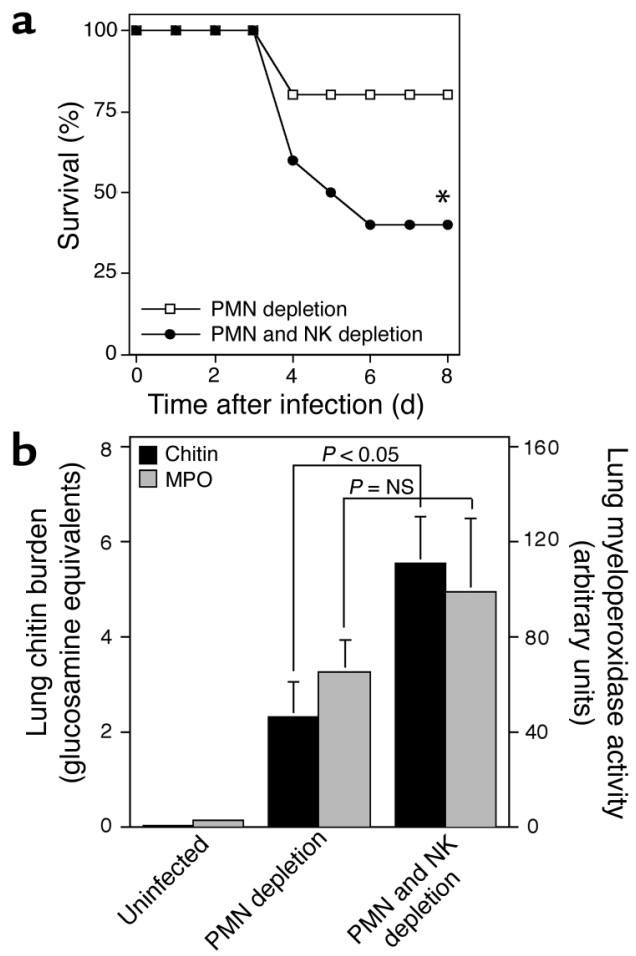


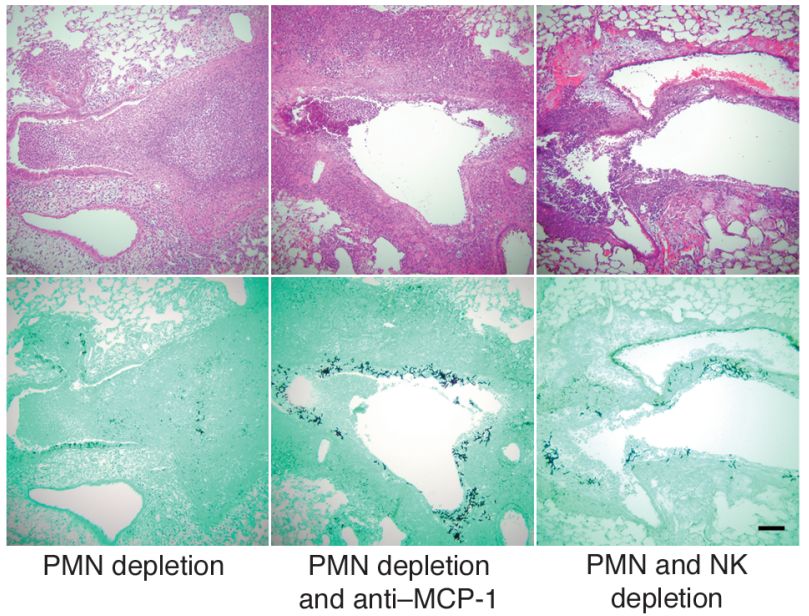

\section{Figure 8}

Effect of NK cell depletion or MCP-1/CCL2 neutralization on lung histology in transiently neutropenic mice with invasive aspergillosis. Representative lung $\mathrm{H} \& \mathrm{E}$ and GMS stains 3 days after inoculation with $A$. fumigatus conidia are shown (original magnification $\times 100$ ). $\mathrm{H} \& \mathrm{E}$ panels in all three groups show areas of intense inflammatory infiltration of leukocytes with some areas of associated necrosis. GMS stains show greater numbers of associated fungal elements in PMN depletion and anti-MCP-1 and PMN and NK depletion groups. PMN depletion indicates RB6-8C5 and PBS; PMN depletion and anti-MCP-1 indicates RB6-8C5-treated mice with MCP-1/ CCL2 neutralization; PMN and NK depletion indicates mice treated with RB6-8C5 and NK1.1.

CCR2 in NK cell influx was to mediate the effects of host MCP-1/CCL2. This hypothesis predicts that in the absence of bioavailable MCP-1/CCL2 in the host, presence or absence of CCR2 on NK cells would not affect their recruitment to the lung; conversely, in the absence of CCR 2 expression on NK cells, availability of MCP-1/CCL2 in the host has no effect on the influx of NK cells. We therefore compared accumulation of CFSE-labeled WT or CCR $2^{-/-}$transferred NK cells in recipients with invasive aspergillosis, with or without immuno-neutralization of MCP-1/CCL2 (Figure 6c). As expected, both MCP-1/CCL2 neutralization and absence of NK cell CCR2 resulted in decreased numbers of labeled NK cells in the lungs. Interestingly, combined neutralization of MCP-1/ CCL2 and absence of CCR2 on transferred NK cells resulted in a further decrement in the accumulation of transferred NK cells in the lungs. This result indicates that the role of MCP-1/CCL2 and NK cell CCR2 in recruiting NK cells to the lungs in this infection is more complex than the exclusive interaction between a single ligand-receptor pair. Specifically, MCP-1/ CCL2 appears to contribute to lung NK cell influx even in the absence of CCR2 expression on NK cells, possibly by inducing the expression of other chemotactic molecules. Similarly, CCR 2 expression by NK cells plays a role in lung NK cell influx even in the setting of MCP-1/CCL2 immuno-neutralization, presumably mediated by other CCR2 ligands.
Effect of NK cell depletion on the outcome of invasive aspergillosis. Given that neutralization of MCP-1/CCL2 resulted in both increased severity of infection and impaired early influx of NK cells into the lungs, we next assessed the role of NK cells in host defenses against invasive aspergillosis by examining the outcome of infection in the setting of NK cell depletion. In survival studies, mice with invasive aspergillosis that had simultaneous depletion of both neutrophils and NK cells had twice the mortality of infected neutrophil-depleted mice with normal NK cells (Figure 7a). In addition, the lung fungal burden in NK-depleted mice was approximately twofold higher in comparison with neutrophil-depleted mice with invasive aspergillosis (Figure $7 b$ ). Since the recruitment of neutrophils into the lungs has been shown previously to correlate strongly with improved outcome in invasive aspergillosis, we also assessed whether the increased severity of infection in NK cell-depleted mice is related to poorer recovery of neutrophil counts or their recruitment to the lungs. On day 3 after challenge with Aspergillus conidia, lung MPO activity, a surrogate for the presence of neutrophils in the lung in this model (19), were not affected by NK cell depletion $(P=0.42)$, indicating that differences in neutrophil influx into the lungs between the two groups did not explain the difference in clearance of the pathogen. Histologically, lungs of neutrophil-depleted mice with either NK cell depletion or MCP-1/CCL2 neutralization had similar degrees of inflammatory infiltration of leukocytes as compared with neutrophil-depleted animals with invasive aspergillosis, but had a greater burden of visible fungal elements (Figure 8).

Given that the lack of either NK cells or MCP-1/CCL2 results in impaired clearance of the A. fumigatus from the lungs, we hypothesized a causal sequence of events: MCP$1 /$ CCL2 mediates the recruitment of NK cells, which in turn contribute to host defense against the pathogen.

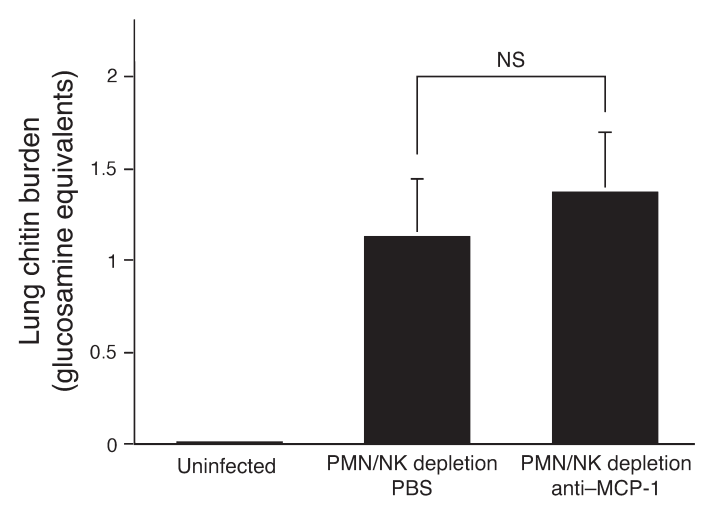

Figure 9

Lung chitin content 3 days after intratracheal challenge with A. fumigatus conidia in neutrophil-depleted mice. Data shown represent mean \pm SEM of $n=6$ per group. PMN/NK depletion PBS indicates mice treated with RB6-8C5, NK1.1, and PBS; PMN/NK depletion anti-MCP-1 indicates mice treated with RB6-8C5, NK1.1, and anti-MCP-1 mAb. 
The competing hypothesis is that while MCP-1/CCL2 recruits NK cells and NK cells contribute to clearing the infection, the beneficial effects of MCP-1/CCL2 in the setting of infection are attributable to a mechanism independent of NK cells. We addressed the latter hypothesis by comparing the lung fungal burden in mice with both NK cell and neutrophil depletion, with or without MCP-1/CCL2 neutralization (Figure 9). In this setting, neutralization of MCP-1/CCL2 had no detectable effect on the lung fungal burden, indicating that the effect of MCP-1/CCL2 on clearance of the pathogen requires the presence of NK cells.

\section{Discussion}

Invasive aspergillosis is an infection with a remarkably poor outcome that almost exclusively afflicts patients with impaired immunity. While neutrophil-mediated antifungal effects are well recognized as critical components of host defense in this infection, other aspects of the response to this infection remain poorly defined. In the current study, we used a previously characterized mouse model of invasive aspergillosis that closely resembles the human disease $(24,27)$ to demonstrate that MCP-1/CCL2 is required for host defense against this pathogen in neutropenic hosts and that this effect is mediated by the early recruitment of NK cells to the lungs.

Our observations provide evidence for a chemokinemediated NK cell effect in pulmonary host defense in this infection. NK cells have been shown to express CCR2 (34-39) and to exhibit in vitro chemotaxis to chemokine ligands (reviewed in ref. 40), including MCP-1/CCL2 (41-45). While these studies have convincingly demonstrated that NK cells are capable of responding to chemokines, the role of this interaction in the complex setting of disease pathogenesis has not been evaluated extensively. In this context, the findings of the present study may have implications beyond lung host defense; the recruitment of NK cells to the lung by MCP-1/CCL2 may, for instance, be a feature of the innate immune response in other diseases.

We found no induction of MCP-1/CCL2 in the lungs of immunocompetent animals challenged with Aspergillus conidia. This observation suggests that in normal hosts, other components of innate immunity are sufficient to clear the pathogen. Conversely, the rapid induction of MCP-1/CCL2 in neutropenic mice with invasive aspergillosis and the increased mortality in the setting of its neutralization indicate a critical role for this mediator in host defense in immunocompromised hosts. It is very possible that other chemotactic factors also play a role in the recruitment of NK cells in response to this infection, as has been demonstrated in response to other pathogens (46). Indeed, we found no attenuation in the late accumulation of leukocyte subsets, including NK cells, in the lungs of animals with on-going MCP-1/CCL2 neutralization; this late influx of leukocytes may represent an appropriate but delayed response to the greater burden of pathogen in lungs of mice with MCP-1/CCL2 neutralization or, conceivably, a nonspecific Ab-mediated effect. Data from the neutralization and receptor knock-out experiments indicate, however, that the early recruitment of NK cells to the lungs is critically dependent on MCP-1/CCL2 and that disruption of this early recruitment results in increased severity of infection. The fact that in the absence of NK cells neutralization of MCP-1/CCL2 had no measurable effect on the severity of infection argues that the contribution of this ligand to host defense in this infection is mediated by NK cells. Our data do not preclude an additional direct or indirect effect of MCP-1/CCL2 on the NK cell activation state or effector functions, independent of its effect on recruitment. An analogous chemokine-mediated activation has, for example, been demonstrated in MIP-1 $\alpha / C C L 3-$ mediated macrophage activation in a bacterial infection (47).

NK cells are best known for mediating immunity against intracellular pathogens, but are also required for normal defenses against several classes of extracellular pathogens, including bacteria (17), parasites (48, $49)$, and yeasts $(50,51)$. The observation that NK cells are essential to host defense against invasive mold infections has not, to our knowledge, been made previously, although NK cell defects have been reported in patients with invasive aspergillosis (52). Our observation may have several significant implications. First, NK cell depletion or dysfunction is a recognized side effect of a number of immunosuppressive treatments associated with invasive aspergillosis (53-56); NK cell defects may thus be a previously unrecognized mechanism predisposing patients to this infection. Second, these results provide a rationale for studying downstream mechanisms by which NK cells mediate antifungal effects in invasive aspergillosis. Last, our results suggest that strategies aimed at increasing the number of available NK cells, enhancing their recruitment, or augmenting their effector functions may represent reasonable therapeutic goals in this infection.

\section{Acknowledgments}

This work was supported in part by NIH grants HL-04220 and HL-73848 and American Lung Association grant RG-005-N.

\footnotetext{
1. Lin, S.J., Schranz, J., and Teutsch, S.M. 2001. Aspergillosis case-fatality rate: systematic review of the literature. Clin. Infect. Dis. 32:358-366.

2. McNeil, M.M., et al. 2001. Trends in mortality due to invasive mycotic diseases in the United States, 1980-1997. Clin. Infect. Dis. 33:641-647.

3. Zlotnik, A., and Yoshie, O. 2000. Chemokines: a new classification system and their role in immunity. Immunity. 12:121-127.

4. Marquez, G., and Martinez, A.C. 2001. Chemokines: the times they are a-changin'. J. Clin. Invest. 107:791-792.

5. Traynor, T.R., et al. 2002. Differential roles of CC chemokine ligand $2 /$ monocyte chemotactic protein-1 and CCR 2 in the development of T1 immunity. J. Immunol. 168:4659-4666.

6. Traynor, T.R., Kuziel, W.A., Toews, G.B., and Huffnagle, G.B. 2000. CCR2 expression determines $\mathrm{T} 1$ versus $\mathrm{T} 2$ polarization during pulmonary Cryptococcus neoformans infection. J. Immunol. 164:2021-2027.

7. Blease, K., et al. 2001. Antifungal and airway remodeling roles for murine monocyte chemoattractant protein-1/CCL2 during pulmonary exposure to Aspergillus fumigatus conidia. J. Immunol. 166:1832-1842.
} 
8. Blease, K., et al. 2000. Enhanced pulmonary allergic responses to Aspergillus in CCR2-/- mice. J. Immunol. 165:2603-2611.

9. Gu, L., et al. 2000. Control of TH2 polarization by the chemokine monocyte chemoattractant protein-1. Nature. 404:407-411.

10. Moore, B.B., et al. 2001. Protection from pulmonary fibrosis in the absence of CCR2 signaling. J. Immunol. 167:4368-4377.

11. Huang, D.R., Wang, J., Kivisakk, P., Rollins, B.J., and Ransohoff, R.M. 2001. Absence of monocyte chemoattractant protein 1 in mice leads to decreased local macrophage recruitment and antigen-specific $T$ helper cell type 1 immune response in experimental autoimmune encephalomyelitis. J. Exp. Med. 193:713-726.

12. Khoo, S.H., and Denning, D.W. 1994. Invasive aspergillosis in patients with AIDS. Clin. Infect. Dis. 19(Suppl 1):S41-S48.

13. Wald, A., Leisenring, W., van Burik, J.A., and Bowden, R.A. 1997. Epidemiology of Aspergillus infections in a large cohort of patients undergoing bone marrow transplantation. J. Infect. Dis. 175:1459-1466.

14. Cohen, M.S., et al. 1981. Fungal infection in chronic granulomatous disease. The importance of the phagocyte in defense against fungi. Am. J. Med. 71:59-66.

15. Gerson, S.L., et al. 1984. Prolonged granulocytopenia: the major risk factor for invasive pulmonary aspergillosis in patients with acute leukemia. Ann. Intern. Med. 100:345-351.

16. Biron, C.A., Nguyen, K.B., Pien, G.C., Cousens, L.P., and Salazar-Mather, T.P. 1999. Natural killer cells in antiviral defense: function and regulation by innate cytokines. Annu. Rev. Immunol. 17:189-220.

17. Jouanguy, E., et al. 1999. IL-12 and IFN-gamma in host defense against mycobacteria and salmonella in mice and men. Curr. Opin. Immunol. 11:346-351.

18. Unanue, E.R. 1997. Inter-relationship among macrophages, natural killer cells and neutrophils in early stages of Listeria resistance. Curr. Opin. Immunol. 9:35-43.

19. Mehrad, B., Strieter, R.M., and Standiford, T.J. 1999. Role of TNF- $\alpha$ in pulmonary host defense in murine invasive aspergillosis. J. Immunol. 162:1633-1640.

20. Kuziel, W.A., et al. 1997. Severe reduction in leukocyte adhesion and monocyte extravasation in mice deficient in CC chemokine receptor 2 Proc. Natl. Acad. Sci. U. S. A. 94:12053-12058.

21. Luo, Y., et al. 1994. Serologic analysis of the mouse beta chemokine JE/monocyte chemoattractant protein-1. J. Immunol. 153:3708-3716.

22. Fleming, T.J., Fleming, M.L., and Malek, T.R. 1993. Selective expression of Ly-6G on myeloid lineage cells in mouse bone marrow. RB6-8C5 mAb to granulocyte-differentiation antigen (Gr-1) detects members of the Ly-6 family. J. Immunol. 151:2399-2408.

23. Lai, L., Alveradi, N., Maltais, L., and Morse, H.C., III. 1998. Mouse cell surface antigens: nomenclature and immunophenotyping. J. Immunol. 160:3861-3868

24. Mehrad, B., et al. 1999. CXC chemokine receptor-2 ligands are necessary components of neutrophil-mediated host defense in invasive pulmonary aspergillosis. J. Immunol. 163:6086-6094.

25. Sentman, C.L., Kumar, V., Koo, G., and Bennett, M. 1989. Effector cell expression of NK1.1, a murine natural killer cell-specific molecule, and ability of mice to reject bone marrow allografts. J. Immunol. 142: $1847-1853$

26. Koo, G.C., and Peppard, J.R. 1984. Establishment of monoclonal antiNK-1.1 antibody. Hybridoma. 3:301-303.

27. Mehrad, B., Moore, T.A., and Standiford, T.J. 2000. Macrophage inflammatory protein- $1 \alpha$ is a critical mediator of host defense against invasive pulmonary aspergillosis in neutropenic hosts. J. Immunol. 165:962-968.

28. Goldblum, S.E., Wu, K.M., and Jay, M. 1985. Lung myeloperoxidase as a measure of pulmonary leukostasis in rabbits. J. Appl. Physiol. 59:1978-1985

29. Kwon-Chung, K., and Bennett, J. 1992. Morphology of Aspergillus species in tissue. In Medical Mycology. Lea \& Febiger. Philadelphia, Pennsylvania, USA. 219-221.

30. Lehmann, P.F., and White, L.O. 1975. Chitin assay used to demonstrate renal localization and cortisone-enhanced growth of Aspergillus fumigatus mycelium in mice. Infect. Immun. 12:987-992.

31. Huffnagle, G.B., Lipscomb, M.F., Lovchik, J.A., Hoag, K.A., and Street, N.E. 1994. The role of CD4+ and CD8+ T cells in the protective inflammatory response to a pulmonary cryptococcal infection. J. Leukoc. Biol. 55:35-42.

32. Kaltreider, H.B., Curtis, J.L., and Arraj, S.M. 1987. The mechanism of appearance of specific antibody-forming cells in lungs of inbred mice after immunization with sheep erythrocytes intratracheally. II. Dosedependence and kinetics of appearance of antibody-forming cells in hilar lymph nodes and lungs of unprimed and primed mice. Am. Rev. Respir. Dis. 135:87-92.

33. Milik, A.M., et al. 1997. Lung lymphocyte elimination by apoptosis in the murine response to intratracheal particulate antigen. J. Clin. Invest. 99:1082-1091.

34. Hodge, D.L., et al. 2002. IL-2 and IL-12 alter NK cell responsiveness to IFN-gamma-inducible protein 10 by down-regulating CXCR3 expression. J. Immunol. 168:6090-6098.

35. Inngjerdingen, M., Damaj, B., and Maghazachi, A.A. 2001. Expression and regulation of chemokine receptors in human natural killer cells. Blood. 97:367-375.

36. Chantakru, S., Kuziel, W.A., Maeda, N., and Croy, B.A. 2001. A study on the density and distribution of uterine natural killer cells at mid pregnancy in mice genetically-ablated for CCR2, CCR5 and the CCR5 receptor ligand, MIP-1 $\alpha$. J. Reprod. Immunol. 49:33-47.

37. Nieto, M., et al. 1998. Roles of chemokines and receptor polarization in NK-target cell interactions. J. Immunol. 161:3330-3339.

38. Sozzani, S., et al. 1997. MCP-1 and CCR2 in HIV infection: regulation of agonist and receptor expression. J. Leukoc. Biol. 62:30-33.

39. Polentarutti, N., et al. 1997. IL-2-regulated expression of the monocyte chemotactic protein-1 receptor (CCR2) in human NK cells: characterization of a predominant 3.4-kilobase transcript containing CCR2B and CCR2A sequences. J. Immunol. 158:2689-2694.

40. Robertson, M.J. 2002. Role of chemokines in the biology of natural killer cells. J. Leukoc. Biol. 71:173-183.

41. Maghazachi, A.A., al-Aoukaty, A., and Schall, T.J. 1994. C-C chemokines induce the chemotaxis of NK and IL-2-activated NK cells. Role for G proteins. J. Immunol. 153:4969-4977.

42. Allavena, P., et al. 1996. Migratory response of human NK cells to monocyte-chemotactic proteins. Methods. 10:145-149.

43. Allavena, P., et al. 1994. Induction of natural killer cell migration by monocyte chemotactic protein-1, -2 and -3 . Eur. J. Immunol. 24:3233-3236.

44. Loetscher, P., Seitz, M., Clark-Lewis, I., Baggiolini, M., and Moser, B. 1996. Activation of NK cells by CC chemokines. Chemotaxis, Ca2+ mobilization, and enzyme release. J. Immunol. 156:322-327.

45. Taub, D.D., Sayers, T.J., Carter, C.R., and Ortaldo, J.R. 1995. Alpha and beta chemokines induce NK cell migration and enhance NK-mediated cytolysis. J. Immunol. 155:3877-3888.

46. Salazar-Mather, T.P., Hamilton, T.A., and Biron, C.A. 2000. A chemokine-to-cytokine-to-chemokine cascade critical in antiviral defense. J. Clin. Invest. 105:985-993.

47. Lindell, D.M., Standiford, T.J., Mancuso, P., Leshen, Z.J., and Huffnagle, G.B. 2001. Macrophage inflammatory protein $1 \alpha / C C L 3$ is required for clearance of an acute Klebsiella pneumoniae pulmonary infection. Infect. Immun. 69:6364-6369.

48. Korten, S., et al. 2002. Expansion of NK cells with reduction of their inhibitory Ly-49A, Ly- 49C, and Ly-49G2 receptor-expressing subsets in a murine helminth infection: contribution to parasite control. J. Immunol. 168:5199-5206.

49. Babu, S., Porte, P., Klei, T.R., Shultz, L.D., and Rajan, T.V. 1998. Host NK cells are required for the growth of the human filarial parasite Brugia malayi in mice. J. Immunol. 161:1428-1432.

50. Djeu, J.Y., Blanchard, D.K., Richards, A.L., and Friedman, H. 1988 Tumor necrosis factor induction by Candida albicans from human natural killer cells and monocytes. J. Immunol. 141:4047-4052.

51. Murphy, J.W., Hidore, M.R., and Wong, S.C. 1993. Direct interactions of human lymphocytes with the yeast-like organism, Cryptococcus neoformans. J. Clin. Invest. 91:1553-1566.

52. Krishnaraj, R., and Svanborg, A. 1993. Low natural killer cell function in disseminated aspergillosis. Scand. J. Infect. Dis. 25:537-541.

53. Ballas, Z.K. 1986. Lymphokine-activated killer (LAK) cells. I. Differential recovery of LAK, natural killer cells, and cytotoxic T lymphocytes after a sublethal dose of cyclophosphamide. J. Immunol. 137:2380-2384.

54. Riccardi, C., Barlozzari, T., Santoni, A., Herberman, R.B., and Cesarini, C. 1981. Transfer to cyclophosphamide-treated mice of natural killer (NK) cells and in vivo natural reactivity against tumors. J. Immunol. 126:1284-1289.

55. Schacter, B., Hansal, S., Arno, J., and LeVine, M.J. 1985. Polymorphic radiation sensitivity of human natural killer activity: possible role of DNA strand breakage. Hum. Immunol. 14:49-58.

56. Porrata, L.F., Inwards, D.J., Lacy, M.Q., and Markovic, S.N. 2001. Immunomodulation of early engrafted natural killer cells with interleukin- 2 and interferon- $\alpha$ in autologous stem cell transplantation. Bone Marrow Transplant. 28:673-680. 